\title{
A design and simulation of a Brazilian Bid Based Short-Term Electricity Market
}

\author{
Felipe Alves Calabria \\ FEUP and $\mathrm{CNPq}$ \\ Fac. de Engenharia da Univ. do Porto \\ Porto, Portugal \\ felipecalabria@gmail.com
}

\author{
João Tomé Saraiva \\ INESC TEC and FEUP/DEEC \\ Fac. de Engenharia da Univ. do Porto \\ Porto, Portugal \\ jsaraiva@fe.up.pt
}

\author{
Ana Paula Rocha \\ LIACC/DEI/FEUP \\ Fac. de Engenharia da Univ. do Porto \\ Porto, Portugal \\ arocha@fe.up.pt
}

\begin{abstract}
In order to overcome a number of difficulties detected on the Brazilian electricity market this paper proposes and tests a bid based short-term market. To simulate the behavior of the hydros in this new market, it was implemented an Agent-Based Model - ABM using the reinforcement QLearning algorithm, Simulated Annealing, and linear programming. In the simulations we used real data from the Brazilian power system encompassing more than $98 \%$ of the total hydro installed capacity and three years of market data. The results indicate that the management of (virtual) reservoirs can be under the responsibility of each hydro, which could save water according to their own risk perception, while it is maintained the current efficiency and security levels. Results also suggest that the final monthly short-term market prices can substantially decrease in comparison with the current prices.
\end{abstract}

Index Terms - Agent-based simulation, Brazilian power system, Q-learning, Virtual reservoirs.

\section{INTRODUCTION}

Several electricity market structures have evolved to ensure free access, guarantee fair competition, foster higher efficiency, and decrease price, while maintaining or improving reliability of operation and security of supply. Although Brazil made significant progresses, its electricity market has certain particularities that contribute to distinguish it from other markets. With a continental interconnected transmission system, a large and growing demand, and a total installed generation capacity around $137 \mathrm{GW}$ [1], from which around $70 \%$ comes from hydropower plants with multiple owners coexisting in hydro cascades, this electricity market has gone through two large institutional and regulatory reforms in the last twenty years. Currently, it contains a number of special processes, mechanisms and instruments that will be detailed in the next paragraphs.

In Brazil, there are two bilateral contract markets: the Free Contracting Environment $-A C L$, where eligible consumers that fit in the characteristic of free consumers or special consumers can choose their supplier; and the Regulated Contracting Environment - $A C R$, designed for distribution companies to buy electricity on behalf of the captive consumers through national public auctions. The $A C R$ operates as the single buyer model where companies sign PPAs - Power Purchase Agreements to build and operate power plants under long-term concessions. The maximum amount of energy that can be traded in the electricity markets is termed the physical guarantee. This is a certificate issued by the Ministry of Mines and Energy that corresponds to the maximum energy production that can be maintained almost continuously over the years. Thus, a power plant can sell part of is physical guarantee in the $A C R$, part in the $A C L$, and still have a fraction to be automatically settled in the short-term market.

When the power plant becomes commissioned, it can perform a seasonal adjustment of the amounts of energy committed through contracts and of its physical guarantee (the total amount of resources to honor the signed contracts). The seasonal adjustment means that generation companies are allowed, once a year, to set the monthly amount of the annual contracted energy and the monthly amount of the physical guarantee. Herewith, regarding the commercial environments, there is an annual "window" to define these amounts. However, the physical dispatch of the power plants is carried out in a centralized way by the Brazilian Independent System Operator - ISO, which doesn't consider the signed contracts when dispatching generation units. The ISO's dispatch goal is to minimize the total operating cost, and this is done using a software package driven by a minimal cost dispatch approach and mainly considering future scenarios of water inflows.

In this scheme, generators are not allowed to decide their own physical generation in order to comply with their contracts, and the ISO decides their outputs without considering the commercial commitments of generators. Thus, if they are not dispatched, they can be exposed to the risk of having to buy electricity at volatile short-term market prices to complete the energy committed in their signed contracts. Because of that, it was implemented a mechanism to share the aforementioned risk among hydros. This mechanism is known as $M R E$ - Mechanism for Energy Reallocation. Shortly, the $M R E$ reallocates energy, transferring the surplus generated from those that produce beyond their monthly allocated 
physical guarantee to those that produce below. Finally, as the $M R E$ is automatically activated, there is no flexibility for hydros to address their exposition risk.

The short-term market (known as $M C P$ ) takes place after the ISO's dispatch. Unlike other markets, the Brazilian shortterm market is not a marketplace where generators are active through a self-dispatch procedure, or generators influence, through their bids, the dispatch schedule. Moreover, the shortterm market price (known as Price for Settlement of Differences or $P L D$ for short) is not the result from the market participant's interaction, but it is one of the outputs of the software package that is run by the ISO. The $M C P$ is simply a mechanism to settle differences between the amounts of electricity committed by contracts and those amounts of electricity that each agent ends up providing/receiving.

Having in mind these characteristics, some problems of this market design are recognized. The conciliation between the commercial commitments and the physical dispatch is not smooth. There is a lack of "trading opportunities" to encourage participants to comply with their contracts (namely related with the unique annual "window" of the seasonalization process). The Brazilian short-term market acts as a mechanism to settle differences rather than a true market, and neither the short-term price nor the dispatch schedule is influenced by the market. The mechanism to share the shortterm risk exposition (MRE) is automatically performed, which imposes a kind of "strait jacket" on the market participants. Finally, inconsistencies in the software package code, the centerpiece of this market design, may cause a huge impact on the sector, affecting the confidence of the market agents.

A solution typically adopted in other electricity markets is the employment of a more market-oriented approach. Designs like that would enable generators to bid, in the short-term, quantities and prices, which could be used to set the market positions and to substitute the seasonalization process and the $M R E$. Nevertheless, there are some concerns to be faced, especially in a power system with a large share of hydros:

- Efficiency of the hydro energy resources. As discussed in [2], it is necessary to coordinate, either through a centralized or decentralized dispatch, the use of the water in the reservoirs in order to take advantage of the all potential stored energy in the cascades. A decentralized dispatch, e.g. a bid based short-term market, can be essentially an instantaneous process and the intertemporal features of river chain operations can not be entirely captured. Moreover, the presence of several owners in the cascades, which is the case of Brazil, endorses a market design based on a centralized dispatch.

- Security of supply. The Brazilian short-term market price $(P L D)$ has an average value of $37.72 € / \mathrm{MWh}$, and the hydro average successful bid price from the national public auctions is $38.16 € / \mathrm{MWh}$ [3]. Nevertheless, the $P L D$ standard deviation is around $55.36 € / \mathrm{MWh}$, which imposes a huge risk to the health of the business, especially regarding cash flow stability. Accordingly, it is advocated the need to have enough incentives and a mechanism to ensure the security of supply. Nowadays, this concern is addressed both via the contracting scheme where the demand must be fully ex-ante contracted and contracts physically backed, and via the ISO dispatch, either through the mechanism of risk aversion implemented into the software package or through a dispatch out of the merit order authorized by CMSE, the federal entity that monitors the supply adequacy.

- Flexibility to comply with contracts. The MRE was created because the ISO's dispatch doesn't consider contracts and hydros cannot influence it. On other hand, if each hydro would decide its own production, MRE would no longer be necessary. Nowadays, every time there is a water shortage, the PLD increases. So, hydros can be displaced by thermal stations in such a way that $M R E$ will not have the extra energy to be shared. In other words, $M R E$ is not able to cover the risk of generators having to buy electricity in the short-term market to complete their commitments. During long periods of water scarcity, this represents a significant negative exposed position. Unfortunately, this situation is happening in Brazil since 2012. This financial issue imposed a "regulation through litigation", and lawsuits mass occurred in such a manner that the entire market settlement was suspended for several months. In the end of 2015 the legislative agenda culminated in the publication of a new federal law and regulatory directive in order to change, for all contracts signed by hydros in both $A C R$ and $A C L$, the allocation risk between generators and consumers.

In other to tackle these problems and concerns it is proposed a new market design aiming at enhancing the flexibility of market agents to comply with their commercial commitments while maintaining the optimized operation of the entire system and the security of supply in the same level. According to these ideas, this paper is organized as follows. Section II explains the proposed virtual reservoir based design, Section III describes the implemented ABM - Agent Based Model simulation, Section IV presents the results and Section $\mathrm{V}$ outlines the main conclusions.

\section{A NeW MARKeT DeSigN}

Based on the concept of energy right accounts as virtual reservoirs, in this new design each hydro is modeled as an agent that has a virtual reservoir representing how much energy is virtually stored in his hydro plant. Hereafter, for each accounting period, each account is fed by the fraction of the total natural affluent energy that flowed to the hydro cascade proportional to the hydro's physical guarantee. Then, the following sequence of events is adopted:

1) The system operator continues his work as it is currently done (running the software dispatch package, as well as other procedures and schemes), and defines the amount of generation for each power plant. This means that it maintains its responsibility in terms of optimizing the use of the resources and keeping the security of supply of the country at the same level;

2) The "remaining demand", which is equal to the total demand minus the total dispatch allocated to the thermal power plants, is obtained for each dispatch period. This value is the total demand to be supplied by hydros; 
3) A bid based hydro short-term market is established for the remaining demand. In this market, hydros have the opportunity to withstand their bilateral commercial commitments while trying to make successful bids. The result of this market is a virtual dispatch with financial settlement purposes. To do that, each hydro agent can bid considering a price between zero and a ceiling price defined by the regulatory agency and a quantity available within his account;

4) The final short-term price is calculated as a weighted average considering the most expensive successful hydro price bid and the variable cost of the last non-hydro resource dispatched by the ISO.

In this new market design two worlds coexist: the real one, associated with the power system considering physical effects, and where the ISO runs the dispatch in a centralized way; and the virtual one, related to the settlement system and with commercial effects, and where hydro agents can virtually bear their contracts. Both worlds simultaneously operate, and the link between them is the real total affluent natural energy that flowed along the hydro cascade in each accounting period. At last, the settlement process will occur considering the successful quantity bid of each participant, and the exposed position will be valued by the new final short-term price. Further explanation about this model is provided in [5].

\section{SimUlation OF THE NEW MARKET}

To simulate the behavior of the hydro plants in this new market design, an Agent-Based Model - ABM was developed using reinforcement the Q-Learning algorithm, a Simulated Annealing (SA) mechanism, and linear programming. According to [6], among the modeling alternatives for bidding strategy analysis, ABM is highlighted because it allows designing complex electricity markets as collections of rulebased agents interacting with one another dynamically and intelligently. An agent using Q-learning is a goal-oriented learner that, for a giving Markov decision process, continually interacts with his environment, receives feedback (rewards or punishments) from it, and searches for the most profitable action considering the past experience. In other words, at each time step $t$ the agent is in some state $s_{t}$, chooses any action $a_{t}$ that is available in state $s_{t}$, receives a corresponding reward $r_{t}$, and moves to a new state $s_{t+l}$. Lastly, the capability of learning to act optimally in Markovian domains by experiencing the consequences of actions is given by a quality function $\mathrm{Q}\left(s_{\mathrm{t}}, a_{\mathrm{t}}\right)$. This function provides the expected utility of taking a given action in a given state, and it is given by (1).

$\mathrm{Q}\left(s_{\mathrm{t}}, a_{\mathrm{t}}\right) \leftarrow \mathrm{Q}\left(s_{\mathrm{t}}, a_{\mathrm{t}}\right)+\alpha \cdot\left\{r_{\mathrm{t}}\left(s_{\mathrm{t}}, a_{\mathrm{t}}\right)+\gamma \cdot \max _{\mathrm{a}} \mathrm{Q}\left(s_{\mathrm{t}+1}, a\right)-\mathrm{Q}\left(s_{\mathrm{t}}, a_{\mathrm{t}}\right)\right\}$

The parameter $\alpha$ is the learning rate, which reflects the degree to which recently learned information will override the old one ( $\alpha$ equal to 0 will make the agent not learn, while equal to 1 will induce the agent to consider only the most recent information), and the parameter $\gamma$ entitles the discount factor that determines the importance of future reinforcements ( $\gamma$ equal to 0 will make the agent myopic by only considering current rewards, while values closer to 1 turn distant rewards more important). Additionally, the expression $\max _{\mathrm{a}} \mathrm{Q}\left(s_{\mathrm{t}+1}, a\right)$ represents the best the agent thinks it can do in state $s_{\mathrm{t}+1}$. In order to adequately balance the capacity not to converge to local optima and the acceleration of the learning process, a Simulated Annealing (SA) mechanism is included into the developed algorithm as detailed in [7].

In brief, to simulate the new market design the algorithm gets from the ISO physical dispatch procedure the total demand to be supplied by non-hydros $\left(\mathrm{Q}_{\mathrm{nh}}\right)$ and by hydros $\left(\mathrm{Q}_{\mathrm{h}}\right)$, and the variable cost of the last non-hydro dispatched unit $\left(\mathrm{PLD}_{\mathrm{nh}}\right)$. It also requires information about ex-ante bilateral contracts $\left(\mathrm{qCE}_{\mathrm{t}}\right)$ for each account period $t$, i.e. the amount of energy that must be endured in the hydro short-term market by the bids. Other input data is as follows: the natural affluent energy that flowed in period $t$ to the hydro cascade $\left(\mathrm{NAE}_{\mathrm{t}}\right)$, from which a fraction is allocated to each hydro giving their physical guarantee (PG); the reservoir capacity (RC), and the virtual reservoir level or energy right account $\left(\mathrm{ERA}_{\mathrm{t}}\right)$ in its initial stage. Bids can be done once respected the following constraints: the quantity bid (qBID) is limited by the ERA balance in each period $t$, and the price bid (pBID) is limited by a maximum regulatory price ( $\mathrm{pBID}$ uplim $\mathrm{t}_{\mathrm{t}}$ ). The bids are sorted by the price, the successful bids are then identified, and the ERA is finally updated.

Agents can offer two bids. The first bid (BID1) addresses the need to comply with bilateral contracts. So, in BID1, among the possible actions, the best offer is either the exact amount in the bilateral contract (if $\mathrm{ERA}_{\mathrm{t}} \geq \mathrm{qCE}_{\mathrm{t}}$ ) or the maximum available amount in order to mitigate the exposed position (if $\mathrm{ERA}_{\mathrm{t}}<\mathrm{qCE} \mathrm{E}_{\mathrm{t}}$ ). The second bid (BID2) aims at allowing the agents to manage their reservoirs in order to optimize the leftover stored energy. That is, agents would be allowed to get extra profit in the short-term market when there is more energy in the reservoir than the one required to comply with the bilateral ex-ante contracts. The Q-learning matrixes are illustrated by Table I (hatched cells represent not allowed actions) and Table II for BID1 and BID2.

\section{TABLE I. BID1: ILLUSTRATION OF THE Q-LEARNING MATRIX}



TABLE II. BID2: ILLUSTRATION OF THE Q-LEARNING MATRIX

\begin{tabular}{|c|c|c|c|c|c|c|c|c|c|c|c|c|c|c|c|}
\hline \multirow{3}{*}{ 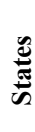 } & January & s1 & & & & & & & & & & & & & \\
\hline & $\cdots$ & $\cdots$ & & & & & & & & & & & & & \\
\hline & December & $s 12$ & & & & & & & & & & & & & \\
\hline \multirow{5}{*}{\multicolumn{2}{|c|}{$\begin{array}{r}\text { pBID }(\% \text { of th } \\
\text { pBID uplim }) \\
\text { qBID }\end{array}$}} & & a1 & a2 & a3 & a4 & a5 & a6 & a7 & $\ldots$ & a23 & a24 & a25 & a26 & a27 \\
\hline & & & 0 & 100 & 0 & 25 & 50 & 75 & 100 & \multirow[b]{2}{*}{$\ldots$} & 0 & 25 & 50 & 75 & \multirow{2}{*}{$\begin{array}{c}100 \\
\%\end{array}$} \\
\hline & & & $\%$ & $\%$ & $\%$ & $\%$ & $\%$ & $\%$ & $\%$ & & $\%$ & $\%$ & $\%$ & $\%$ & \\
\hline & & & & 0 & \multicolumn{5}{|c|}{$20 \%$. ERAaBID1 } & $\ldots$ & \multicolumn{5}{|c|}{$100 \%$. ERAaBID 1} \\
\hline & & & \multicolumn{13}{|c|}{ Actio } \\
\hline
\end{tabular}

Then, the reward for BID1 is calculated using equation (2) and for BID2 according to equation (3). 
rewardBID1 $1_{t}=q B I D 1 s u c_{t}-q C E_{t}$

rewardBID $2_{\mathrm{t}}=$ factor $_{\text {goalt }} *\left(\mathrm{qBID} 2 \mathrm{suc}_{\mathrm{t}}+\mathrm{ERAaBID} 2_{\mathrm{t}}\right) * \mathrm{PLD}_{\text {finalt }}$

In these equations, qBID1suc $\mathrm{c}_{\mathrm{t}}$ and qBID2 suc $_{t}$ are the successful bid quantities, ERAaBID $2_{t}$ is the leftover energy in the reservoir after BID2 in period $t$, and the factor goalt $_{\text {is }}$ the adjustment factor concerning the reservoir level goals. The value of the factorgoalt is obtained solving a linear programming problem aiming to avoid spillage and to maximize profits on the hydro short-term market. The decision of considering qBID2 suc $_{t}$ more important than ERAaBID2 ${ }_{t}$ (i.e. use the water stored) and vice versa (save the water for future usage), is guided by the factor goalt $_{\text {. If the agent }}$ offers a large quantity of energy when qBID should be low, the current level of the reservoir will be away from the goal level (defined by the linear programming), and the reward will penalize the agent through the factor ${ }_{\text {goalt }}$.

Before running a real case, the Brazilian electricity market, it was developed a validation process for the entire algorithm. During this process, the verification of the computer program, written in Matlab, was performed several times, distinct values of the parameters used in the aforementioned Q-learning, Simulate Annealing and linear programming algorithms were tested, and several convergence criteria were verified. Finally, questions regarding the collective and individual rationality of the ABM agents were adequately addressed.

\section{RESULTS}

A major effort was undertaken to use in the simulations accurate and comprehensive data from Brazil. A total of 125 hydro power plants were simulated, which represents more than $98 \%$ of the total hydro installed capacity in the country. Besides the data about hydros, it was also used market data embracing 3 years, 2012, 2013 and 2014.

For each year, the simulations considered three levels of ex-ante contract $(95 \%, 50 \%$ and $0 \%)$, two types of bilateral contracts' seasonalization ( $N A E$, in which the annual amount is allocated bearing in mind weights determined by the water inflow; and FLAT, where the annual energy committed is monthly distributed in equal amounts), and two types of ceiling price rules ( $P L D n h$, i.e. equivalent to the variable cost of the last non-hydro station dispatched; and Fixed, a fixed ceiling price equal to the annual ceiling price established by the national regulatory agency). This said, 12 scenarios were performed in each year. Each simulation takes about 12 minutes to converge, corresponding to around 1.100 years of learning to reach the final results. The convergence criterion requires that the values of the Q-learning matrix built in a given iteration regarding the previous one do not change more than $0,5 \%$. The learning rate $\alpha$ is equal to 0,9 in both BID1 and BID2, and discount factor $\gamma$ is equal to 0 in BID1 and equal to 1 in BID2.

As can be seen in Tables III, IV and V, during all the years the annual PLD resulting from the simulation is lower (about $60 \%$ ) than the real PLD. The fourth scenario (highlighted in the three Tables), given its characteristic, is the most representative scenario of the current Brazilian market, and its results show that new monthly prices are about $70 \%$ lower in two trimesters, and 50\% lower in the last trimester.
TABLE III. YEAR 2012: SimULATED PLD versus ACtUAL PLD

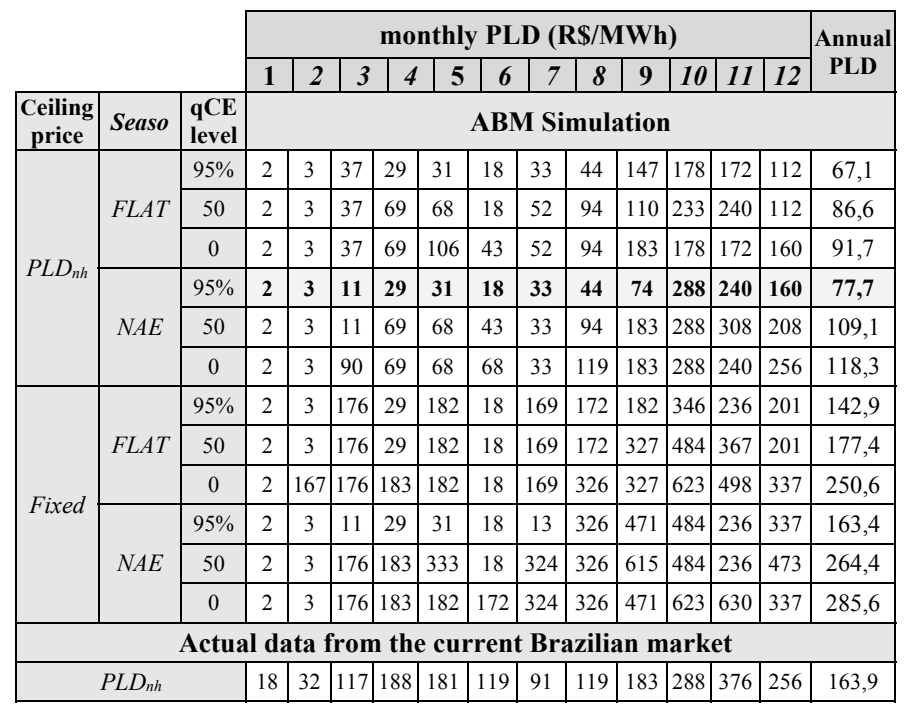

TABLE IV. YEAR 2013: SimULATED PLD VERSUS ACTUAL PLD



TABLE V. YEAR 2014: SiMULATED PLD VERSUS ACTUAL PLD

\begin{tabular}{|c|c|c|c|c|c|c|c|c|c|c|c|c|c|c|c|}
\hline & \multicolumn{12}{|c|}{ monthly PLD (RS/MWh) } & \multirow{2}{*}{\begin{tabular}{|l} 
Annual \\
PLD
\end{tabular}} \\
\hline & & & 1 & 2 & 3 & 4 & 5 & 6 & 7 & 8 & 9 & 10 & 11 & 12 & \\
\hline eiling & Seaso & & \multicolumn{13}{|c|}{ ABM Simulation } \\
\hline \multirow{6}{*}{$L D_{n h}$} & \multirow{3}{*}{ FLAT } & $95 \%$ & 82 & 314 & 216 & 215 & 215 & 116 & \begin{tabular}{|l|}
181 \\
\end{tabular} & 368 & 370 & 270 & 544 & 212 & 8,4 \\
\hline & & $50 \%$ & 82 & 314 & 216 & 215 & 215 & 116 & 278 & 368 & 370 & 518 & 544 & 212 & 87, \\
\hline & & $0 \%$ & 156 & 447 & 216 & 215 & 331 & 116 & 278 & 596 & 609 & 766 & 413 & 309 & 70,9 \\
\hline & \multirow{3}{*}{$N A E$} & $95 \%$ & 82 & 181 & 216 & 215 & 215 & 116 & 181 & 368 & 489 & 518 & 413 & 212 & 67,1 \\
\hline & & $50 \%$ & 82 & 181 & 216 & 215 & 215 & 116 & 181 & 368 & 489 & 642 & 674 & 212 & 299,1 \\
\hline & & $0 \%$ & 82 & 314 & 216 & 215 & 215 & 116 & 278 & 596 & 609 & 766 & 413 & 212 & 35,9 \\
\hline \multirow{6}{*}{ Fixed } & \multirow{3}{*}{$F L A T$} & $95 \%$ & 82 & 488 & 216 & 215 & 215 & 116 & 181 & 254 & 250 & 536 & 550 & 212 & 276,1 \\
\hline & & $50 \%$ & 243 & 642 & 216 & 215 & 215 & 116 & 181 & 518 & 385 & 403 & 683 & 212 & 335, \\
\hline & & $0 \%$ & 243 & 334 & 216 & 362 & 355 & 116 & 462 & 386 & 385 & 403 & 416 & 545 & 35,3 \\
\hline & \multirow{3}{*}{$N A E$} & $95 \%$ & 82 & 181 & 216 & 215 & 215 & 116 & 181 & 386 & 520 & 403 & 683 & 212 & 284,1 \\
\hline & & $50 \%$ & 82 & 488 & 216 & 215 & 215 & 116 & 321 & 518 & 520 & 669 & 550 & 212 & 343,5 \\
\hline & & $0 \%$ & 403 & 181 & 364 & 215 & 215 & 116 & 321 & 386 & 520 & 536 & 683 & 345 & 57 \\
\hline \multicolumn{16}{|c|}{ Actual data from the current Brazilian market } \\
\hline & $L D$ & & 375 & 714 & & 758 & 680 & 361 & 570 & 710 & 729 & 766 & 805 & 5 & 653,5 \\
\hline
\end{tabular}


Figures 1 and 2 provide more insight regarding the hydro's operation. For the Brazilian four largest hydros (in terms of installed capacity) with reservoir, during 2014 (the most recent year) and for scenario no. 4, these Figures show the best strategy chosen to sustain the bilateral contracts (BID1) and to seek profit from the leftover energy (BID2).

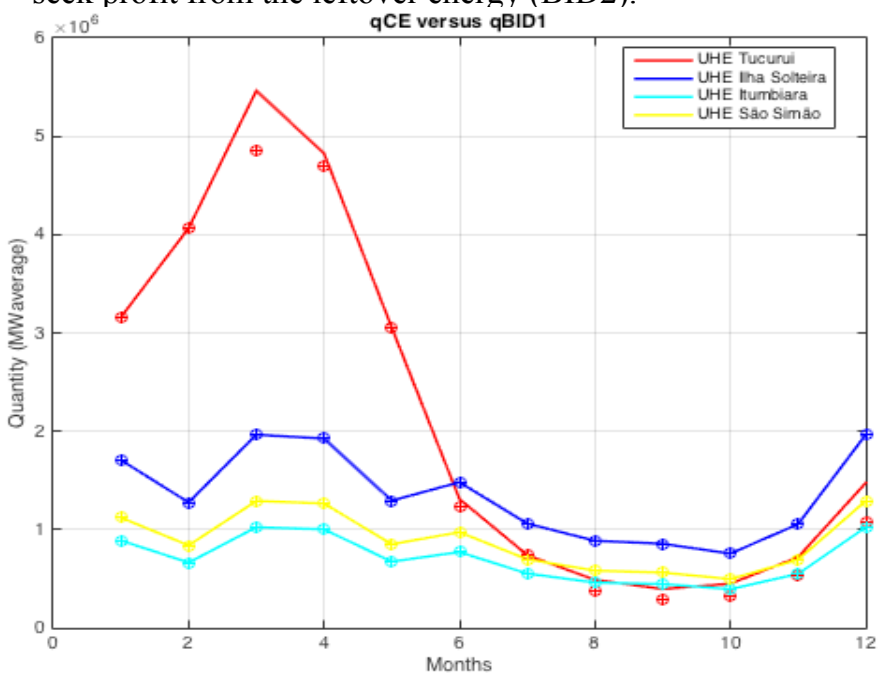

Figure 1. qBID1: Sustaining the bilateral contracts (scenario no. 4)

As can be noticed in Figure 1 (lines are qCEs and dots are qBID1s), hydros are able to learn to sustain bilateral contracts. This is done by choosing qBID1 as close as possible to qCE. Only Tucuruí and Luiz Gonzaga, during some months, cannot monthly endure $100 \%$ of the bilateral contract by qBID1. This happens because Tucuruí and Luiz Gonzaga started the year with $44,23 \%$ and $42,02 \%$ of the reservoir, respectively. Additionally, in 2014 water inflows were 11,48\% below the average of a historical series of 84 years. So, given these circumstances, there is nothing that they can do to avoid this.


Figure 2. qBID2: Best strategy to use the leftover energy (scenario no. 4)

Regarding the second bid, Figure 2 presents a 3D "map" where the vertical axe contains the value of each cell of the Qlearning matrix, and the other axes have the actions and the states. So, the top of the "mountains" represents the best action to be taken in each month. In Tucuruí and Luiz Gonzaga we can note a "mountain range" located at the action a1. Action a1 means that the company should offer a qBID2 and a pBID2 equal to zero, and this action is chosen whenever a hydro doesn't have water to use in BID2. So, these two sets of graphics can be seen as complementary to each other.

\section{CONCLUSION}

The entire algorithm combines a goal-oriented scheme built through the mentioned linear programming, a goaloriented learner from the Q-learning, and Simulated Annealing to balance exploring and exploiting the reinforcement learning process. The obtained results indicate that agents are capable to act on their own in order to seek the best strategist to sustain their bilateral contracts and to manage their reservoirs aiming at optimizing the leftover energy.

The obtained results also reveal that monthly $P L D$ greatly decrease in comparison with the current real price. Although the new $P L D s$ could be equal to the ceiling price, they are, at maximum, at $50 \%$ this value. This is explained considering two elements: competition among hydros to endure bilateral contracts and contracting scheme where loads must be fully ex-ante contracted. Both elements are linked. The contracting scheme pushes hydros to sign contracts and, later on, competition in short-term market forces them to bid at lower prices to deliver the contracted energy.

Finally, from a broad perspective, the proposed market design maintains the current levels of efficiency and security, while increasing the level of flexibility regarding the commercial behavior of the agents. In the Brazilian case, this flexibility is achieved by replacing the $M R E$ and the seasonalization processes by a virtual reservoir model. As a consequence, the management of (virtual) reservoirs is under the responsibility of each hydro, which could (virtually) save water according to their own risk perception. In doing so, the operation of the physical system by the ISO is not affected, ensuring the efficiency of the hydro cascade operation and maintaining the current level of the security of supply.

\section{REFERENCES}

[1] ANEEL - Brazilian Electricity Regulatory Agency. Database of electricity generation [Online]. Available: http://www.aneel.gov.br

[2] A. Philpott, Z. Guan, J. Khazaei, G. Zakeri, "Production inefficiency of electricity markets with hydro generation," Utilities Policy, vol. 18, pp. 174-185, Dec. 2010.

[3] CCEE - Electric Power Commercialization Chamber. Prices. [Online]. Available: http://www.ccee.org.br

[4] Li G., Shi J., Qu X. "Modeling methods for GenCo bidding strategy optimization in the liberalized electricity spot market: A state-of-the-art review". Energy, vol. 36, pp. 4686-4700, 2011.

[5] F. Calabria; J. T. Saraiva; J-M. Glachant. "Enhancing flexibility and ensuring efficiency and security: Improving the electricity market in Brazil". Policy Brief. EUI - European University Institute, RSCAS Robert Schuman Center for Advanced Studies, FSR - Florence School of Regulation. Issue 2014/07, Dec 2014.

[6] A. Weidlich; D. Veit. "A critical survey of agent-based wholesale electricity market models". Energy Economics, 30, pp. 1728-1759, 2008.

[7] M. Guo; Y. Liu; J. Malec, "A new Q-learning algorithm based on the metropolis criterion", IEEE Transactions on Systems, Man, and Cybernetics, Part B, vol. 34, n. 5, pp. 2140-2143, Oct. 2004. 